\title{
HAPTIC HUMAN INTERFACES FOR ROBOTIC TELEMANIPULATION
}

\author{
Emil M. Petriu, Pierre Payeur, and Ana-Maria Cretu \\ School of Information Technology and Engineering \\ University of Ottawa \\ 800 King Edward, Ottawa, ON, Canada, K1N 6N5 \\ \{petriu, ppayeur, acretu\}@site.uottawa.ca \\ http://www.site.uottawa.ca
}

\begin{abstract}
Recent investigation in haptic man-robot interaction suggests that there are ultimately only two topical tactile feedback generation modalities for haptic human interfaces. These allow the human operator to handle either (i) temporary virtual reality-based material replicas of the local geometric and/or force profile at the contact areas of an unlimited set of generic objects that could virtually be handled during the manipulation, or (ii) permanent material replicas of a limited set of typical objects. In this paper, the two modalities are analyzed and examples of tactile human interfaces developed by the authors for telerobotic blind tactile exploration of objects, and for telerobotic hapto-visual stylus-style tool manipulation are presented to illustrate the proposed approaches. The necessary modelling of the elastic properties of $3 D$ objects from experimental tactile and range imaging data is also presented using a neural network architecture that becomes an important component of the haptic interface.
\end{abstract}

Keywords: Haptics, tactile feedback, human interfaces, deformable objects, elastic behaviors, telerobotics, neural networks.

\section{INTRODUCTION}

While discussing human perception mechanisms, Sekuler and Balke [1] eloquently stated that

... whether exploring gross or small details,

the hand and the finger pads convey the most useful tactile information about objects. In this respect, the hand is analogous to the eye's fovea, the region of retina associated with keen visual acuity. There is, however, a flaw in this analogy: fovea vision is most acute when the eye is relatively stationary, but touch acuity is best when the fingers move of the object of regard.

Human haptic perception is the result of a complex investigatory dexterous manipulation act involving two distinct sensing components: (i) tactile, or cutaneous, information from touch sensors which provide data about contact force, local geometric profile, texture, and temperature of the touched object-area, and (ii) kinesthetic information about the positions and velocities of the kinematic structure (bones and muscles) of the hand [2]. The highest density of cutaneous sensors is found in finger pads. Force information is mostly provided by sensors on muscles, tendons and joints.

The potential of the emergent haptic perception technologies is significant for applications requiring object telemanipulation. such as: (i) robot-assisted handling of materials in industry, hazardous environments, high risk security operations, or difficult to reach environments [3], (ii) telelearning in hands-on virtual laboratory environments for science and arts [4], (iii) telemedicine and medical training simulators [5]. Telerobotic dexterous manipulation in changing and unstructured environments combines the low-level robot computer control with the higher-level perception and task planning abilities of a human operator equipped with adequate human interfaces [6].

Haptic and visual perception modalities complement each other [7]. The resulting multisensor perception allows human operators to have a telepresence experience virtually identical with what they would have had while manipulating real physical objects.

Robotic dexterous manipulation is an objectoriented act requiring not only specialized robotic hands with articulated fingers but also tactile and kinesthetic sensors for the precise control of the forces and motions exerted on the manipulated object [6], [8].

Recently became commercially available a 24 movement robot dexterous hand providing a detailed direct mapping of human hand structure and 
functions, [9], [10]. It is actuated by 40 compliant "air muscles which allows for the manipulation of soft or fragile objects".

In his state-of-the-art review [11], McDonald surveys a wide breadth of Human Machine Interfaces with the declared aim of identifying those "that work right for the people that the robots are built for." The report's conclusions are that a more coordinated effort is needed and added rigor is called for in order to develop adequate human interfaces which will allow intelligent machines to behave and interact with humans in a more humanlike way.

In a more recent survey covering a 20 year period from the late 1980s to early 2000s [12], BenaliKhoudja et al. provide a thorough review of the tactile human interface and their applications to teleoperation, telepresence, sensory substitution, 3D surface generation, Braille systems, laboratory prototypes, and games.

Cutaneous tactile perception is essential for the dexterous manipulation of the objects [6], [13]. The cutaneous performance of the haptic human interfaces should meet the requirements summarized in [12]: $1 \mathrm{~mm}$ distance between micro-actuators, 300 $\mathrm{Hz}$ bandwidth for each micro-actuator, and a threshold of $0.5 \mathrm{MPa}$. It is of primary importance to couple the vibrating mechanical actuators with thermal actuators that will cover an interval from 10 to 45 deg. C.

However, while there are commercial haptic human interfaces able to provide a full kinesthetic component of the haptic perception, there are no commercial interfaces able to provide a comparatively rich cutaneous component. The commercial interfaces which we are aware of have small vibro-tactile stimulators on the back of each finger and on the palm of the interface letting the human user know that an individual finger or the palm has made contact with the manipulated virtual object. However, this does not allow discriminating between specific locations on the alerted finger or palm, or allows any fingertip touch experience.

This paper discusses the basic generation principles for the local geometric and force profile components of the tactile feedback provided by the haptic human interfaces. This approach allows for the design of specialized haptic human interfaces that are optimized for typical haptic manipulation tasks. Examples of tactile human interfaces developed by the authors for telerobotic blind tactile exploration of objects and for telerobotic haptovisual stylus-style tool manipulation are presented to illustrate the proposed design approach.

The paper concludes with the description of a neural network hapto-visual modeling technique that allows the capture, storage, and rendering in real- time of the complex elastic properties of 3D objects from experimental tactile and range imaging data.

\section{TACTILE FEEDBACK GENERATION IN HAPTIC HUMAN INTERFACES}

Haptic perception implies direct contact between the operator's hand and the manipulated object. This means that any haptic human interface should allow the human operator to touch either (i) temporary virtual reality-based material replicas of the local geometric and/or force profile at the relatively small contact areas that are virtually being touched at any given moment during the manipulation of an unlimited set of generic objects, or (ii) permanent material replicas of a limited set of whole typical objects.

\section{A. TACTILE HUMAN INTERFACES USING TEMPORARY REPLICAS OF LOCAL GEOMETRIC OBJECT PROFILE}

An early tactile human interface providing a temporary replica of the local geometric and/or force profile at the contact areas of the object that are virtually being touched was proposed in 1982 by Petriu et al. [14] as part of a multimodal controlled (video, audio, and haptic) telerobotic system, Fig. 1.

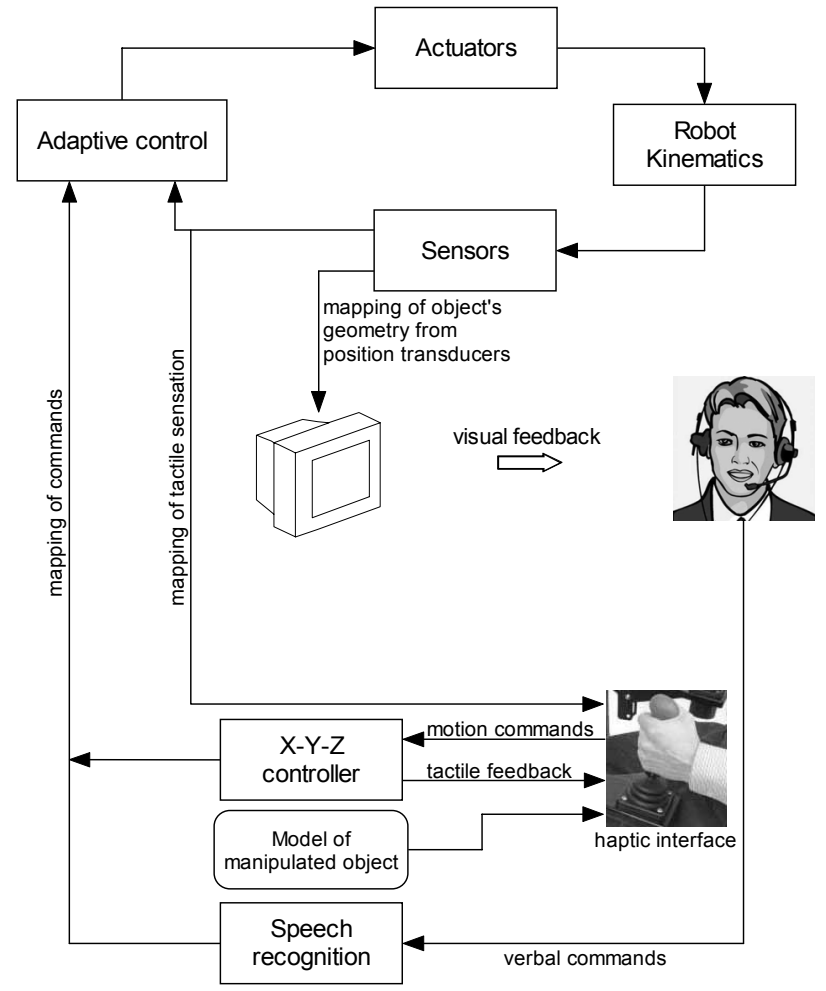

Fig. 1 - Illustration of the 1982 vintage multimodal controlled telerobotic system.

The handheld haptic human interface, Fig. 2b, provides tactile feedback allowing the human teleoperator to feel the local geometric profile 
measured by the tactile sensor arrays in the robot fingers. It consists of two arrays of 23 spring-loaded electromagnetic vibrators, each array having the same dimensions and arrangement pattern as the linear variable-differential transformer (LVDT) tactile sensor arrays in the robot fingers. The two electromagnetic vibrator arrays are positioned on the opposing faces of a $50 \mathrm{~mm}$ by $18 \mathrm{~mm}$ by $24 \mathrm{~mm}$ parallelepiped.

Tactile sensor arrays placed on the two fingers of the robotic manipulator measure the local geometric profile of the object areas touched by the robot's fingers, Fig. 2a. Each tactile sensor array consists of 23 individual LVDT displacement sensors tightly arranged $6 \mathrm{~mm}$ apart in three rows, of 8,7 , and respectively 8 sensors. The tactile sensor arrays covers a $42 \mathrm{~mm}$ by $10.4 \mathrm{~mm}$ contact area.

Another haptic human interface providing a temporary material replica of the local geometric and force profile at the contact areas of the object that are virtually being touched was proposed in 1992 by Petriu et al. [15] as part of an integrated vision and tactile sensing system for model-based object recognition applicable to space station special purpose dexterous manipulator [16].

The biology-inspired robot haptic perception system, shown in Fig. 3, consists of a robot "finger", an instrumented passive-compliant wrist-joint and a tactile probe array [16]. Position sensors placed in the robot joints and on the instrumented passivecompliant wrist provide the kinesthetic information. The compliance of the wrist joint allows the tactile sensor to align itself with the touched object surface and thus to increase the local cutaneous information extracted during the active exploration process under

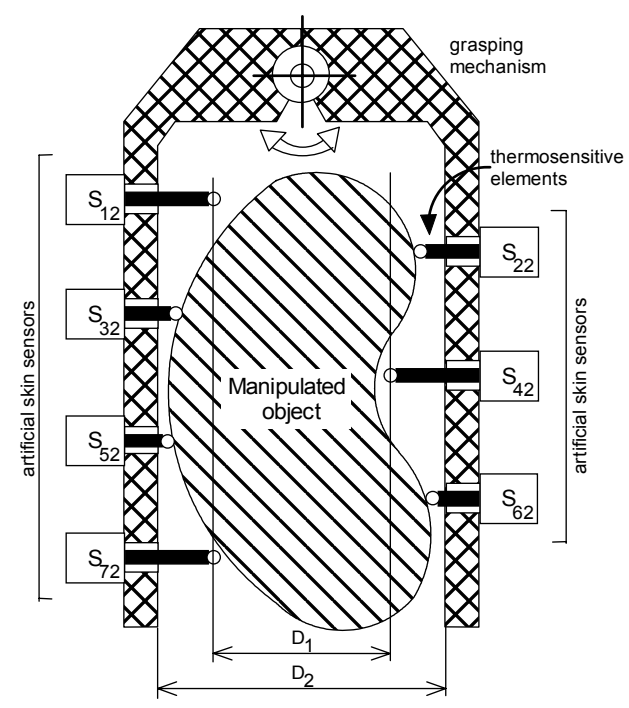

a) the force provided by the robot. The approach has been recently extended for telerobotic blind tactile recognition of tiny objects such as screw head localization and type determination in automated industrial applications [17].

The tactile probe, Fig. 4, consists of a 16-by-16 matrix of Force Sensing Resistor (FSR) elements spaced $1.58 \mathrm{~mm}$ apart on a $6.5 \mathrm{~cm}^{2}$ (1 sq. inch) area and an elastic overlay that provides a geometric profile-to-force transduction function [18]. It operates as an integrated tactile sensor with its own microcontroller to sense compression forces through the elastic overlay [17]. When coupled with a vision guidance system, it can provide the cutaneous component that can be reproduced to the human operator through a tactile feedback mechanism.

The tactile human interface, Fig. 5, allows a human teleoperator to experience a direct tactile feeling of the object profile measured by the robot's tactile sensor. It consists of an 8-by-8 array of electromagnetic vibrators covering a $6.5 \mathrm{~cm}^{2}$ contact area. Each stimulator corresponds to a 2-by-2 "taxel" (i.e. tactile pixel) window in the tactile sensor array. The vibrotactile stimulator is used as binary device which is activated when at least two out of four "taxels" are on.

Before being used for the actuation of the tactile human interface, the tactile sensor data are median filtered in order to remove the measurement noise while preserving the edges in the tactile image. Experiments have shown that it is possible to increase the resolution of the reconstructed local geometric profile by using a pseudo-random selection of the electromagnetic vibrators which are activated sequentially.

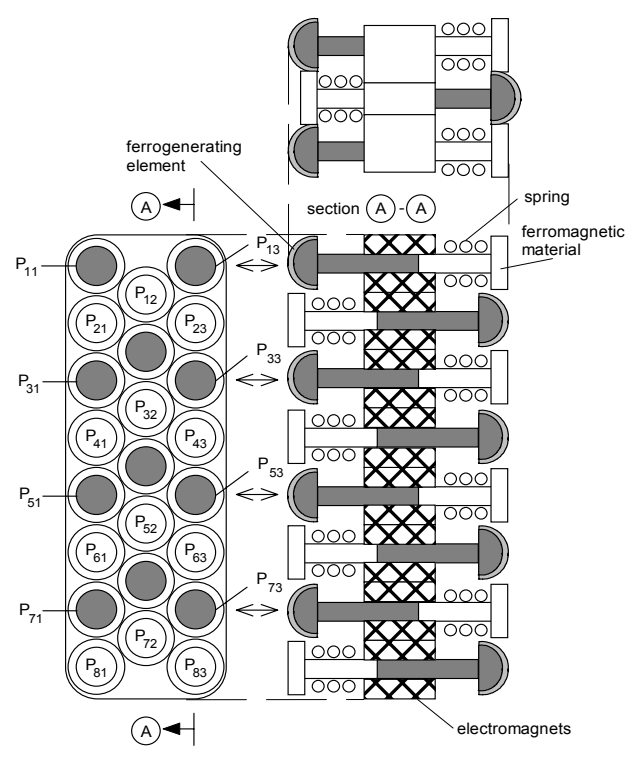

b)

Fig. 2 - Illustration of a) the tactile robot sensor, and b) the corresponding tactile human interface of the telerobotic system, as introduced in [14]. 


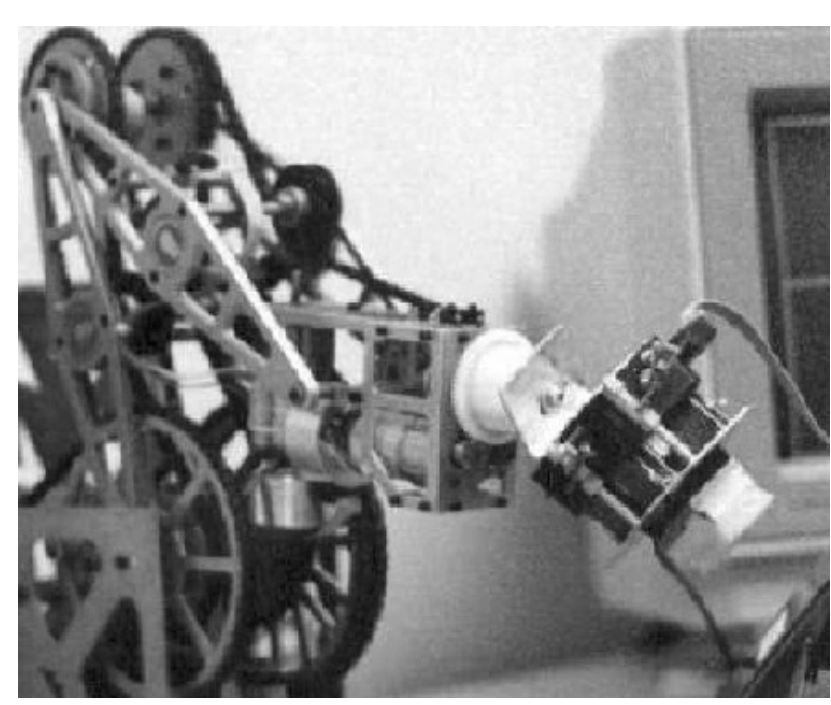

Fig. 3 - Robotic manipulator equipped with an instrumented passive-compliant wrist-joint and a 16-by-16 tactile probe.

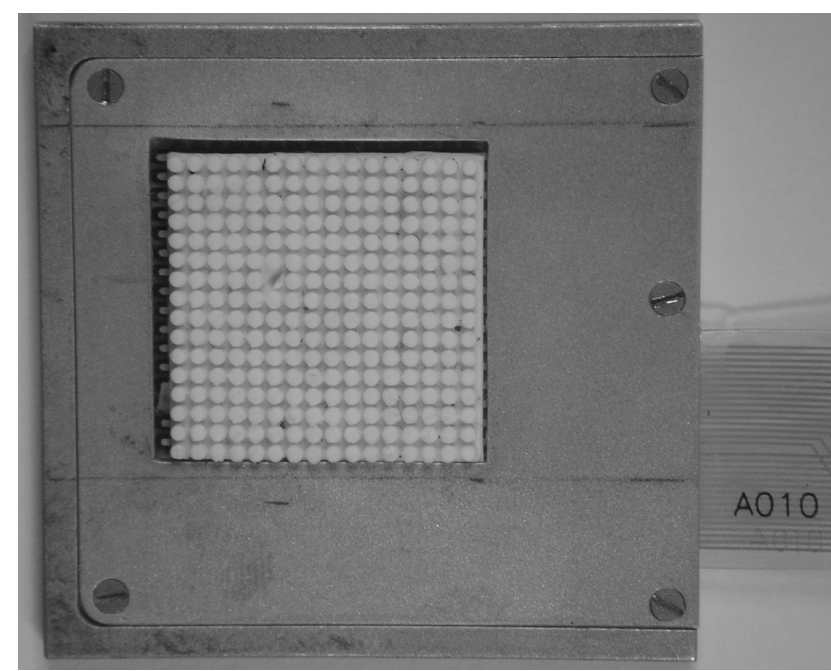

Fig. 4 - 16-by-16 tactile sensor array.

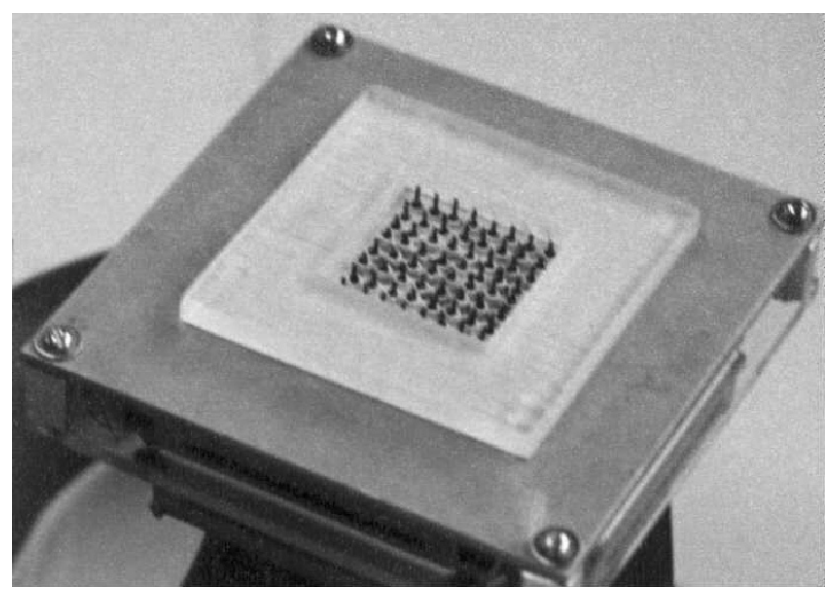

Fig. 5 - Tactile human interface consisting of an 8-by-8 array of electromagnetic vibrators.

\section{B. HAPTIC HUMAN INTERFACES USING PERMANENT REPLICAS OF WHOLE TYPICAL OBJECTS.}

There are many telemanipulation applications which involve the handling of a limited set of objects, usually tools. Stylus type tools such as pens, surgeon knifes, screwdrivers, probing rods, etc, are frequently used in dexterous manipulation applications.

Fig. 6 shows a robot manipulated rod probing the elastic properties of an object. The elastic behaviour at any given point $\left(x_{p}, y_{p}, z_{p}\right)$ on the object surface can be described by Hooke's law:

$$
\left\{\begin{array}{ccc}
\sigma_{p}=E_{p} \cdot \varepsilon_{p} & \text { if } & 0 \leq \varepsilon_{p} \leq \varepsilon_{p_{\max }} \\
\sigma_{p}=\sigma_{p_{\max }} & \text { if } & \varepsilon_{p_{\max }}<\varepsilon_{p}
\end{array}\right.
$$

where $E_{p}$ is the modulus of elasticity, $\sigma_{p}$ is the stress, and $\varepsilon_{p}$ is the strain along the normal direction, and $\sigma_{p \max }$ corresponds to the elastic range. However, Hooke's law applies well for a number of materials (known as Hookean materials) such as steal or aluminium. But for other materials, like rubber, it cannot capture the dependency on phenomena like temperature and loading rate. A nonlinear mapping must then be defined from experimental measurements.

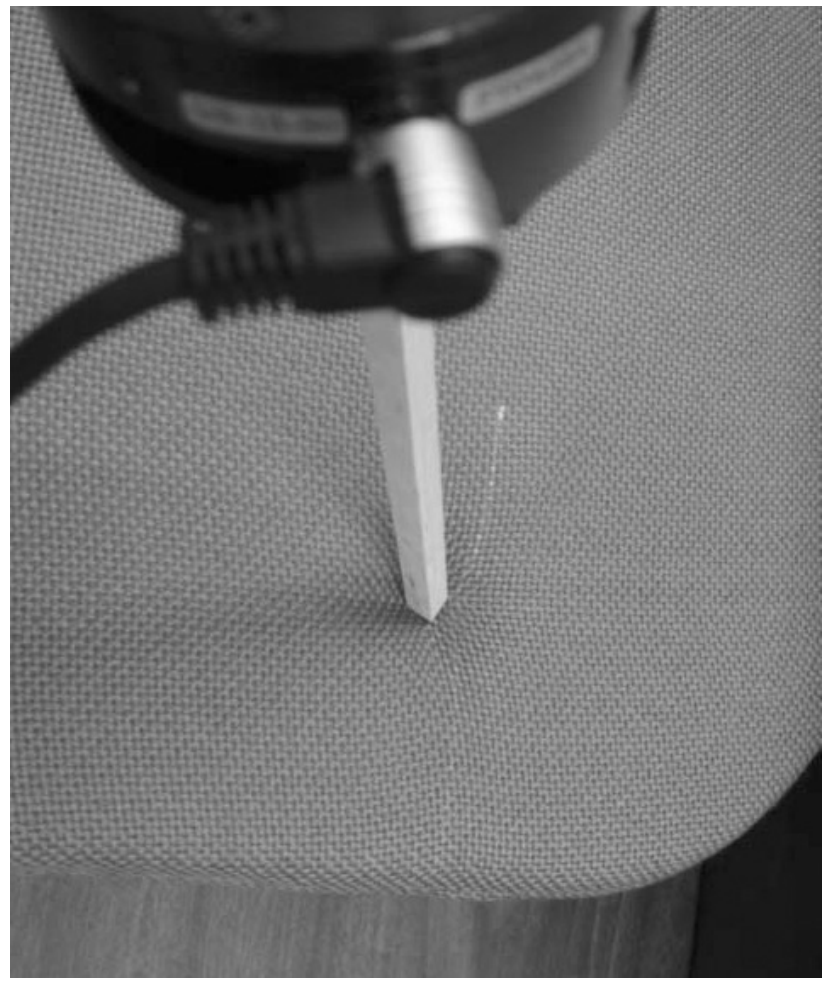

Fig. 6 - Force-torque sensor measuring the interaction force and torque at the point of contact between the robot manipulated probing rod and the object. 
Recovery of the elastic material properties requires touching each point of interest on the explored object surface and then conducting a haptovisual measurement of the strain-stress relation on each point for a sequence of magnitudes of the normal force applied by the robot manipulated probing rod [19].

The force torque-sensor at the robot's end effector, Fig. 6, allows measuring the 3 degree-offreedom (DOF) components of the force vector and respectively the 3 DOF torque components at the point of contact between the probing rod and the explored object [20]. The corresponding geometric profile of the object area touched by the probing rod is measured by a laser range-finder system as shown in Fig. 7 along both of the perpendicular directions to obtain a $2 \mathrm{D}$ deformation map.

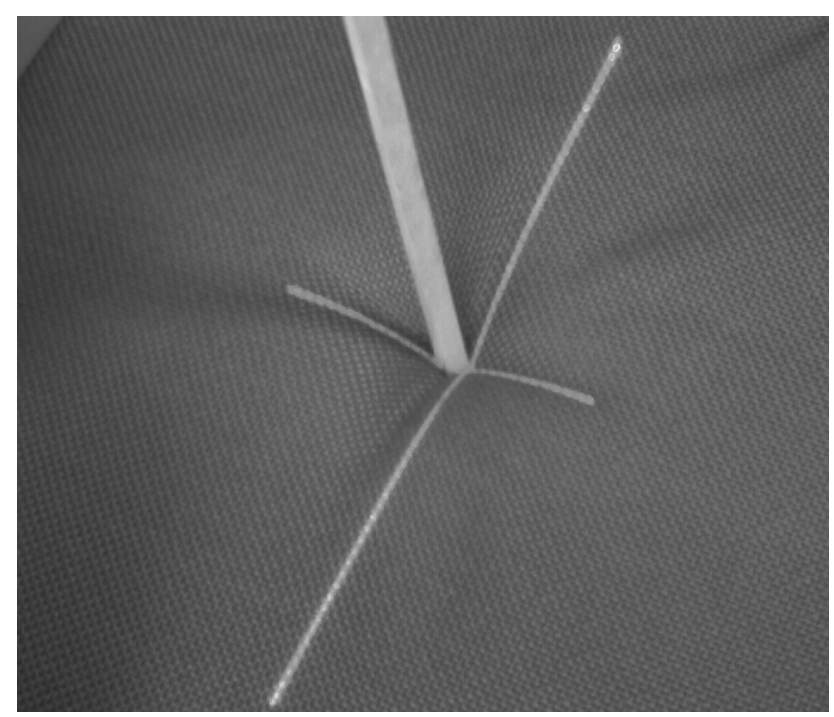

Fig. 7 - Laser range-finder based recovery of the geometric profiles in an area around the contact point between the probing rod and the object.

Fig. 8 shows the geometric profiles measured by the laser range-finder system respectively on the horizontal and on the vertical direction at the point of contact. Assuming symmetrical elastic properties of the deformable object, these two perpendicular profiles are swept circularly and interpolated to compute a surface map of the deformation around the probing point, as shown in Fig. 9.

As expected, the laser range-finder cannot measure the coordinates precisely at the point of contact which is actually obstructed by the probing rod. These coordinates are estimated based on the horizontal and vertical geometric profile measurements for the area immediately close to the point of contact. Knowledge about the rod's length and the pose of the robot's end effector handling the probe with respect to the surface of the object is also taken into account.

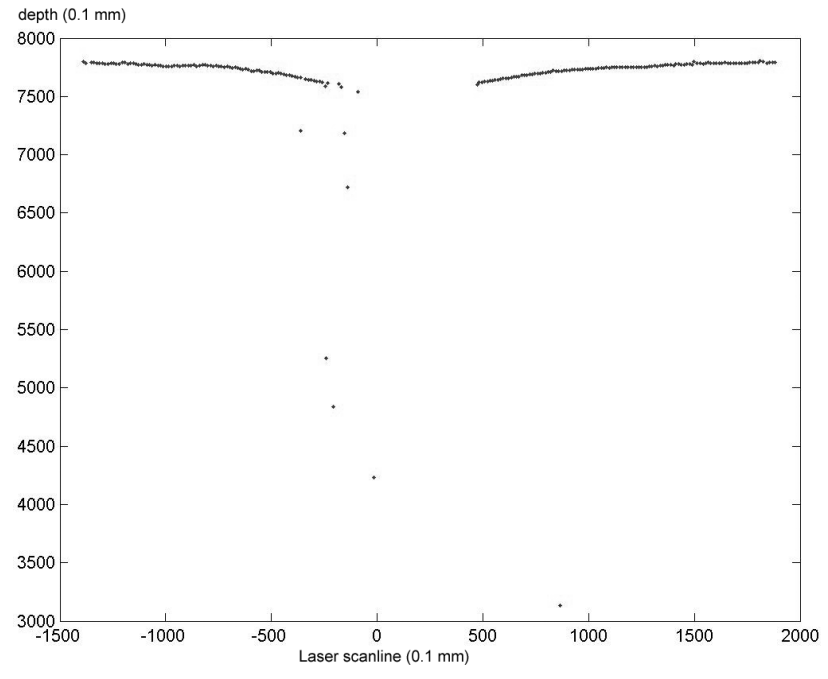

a)

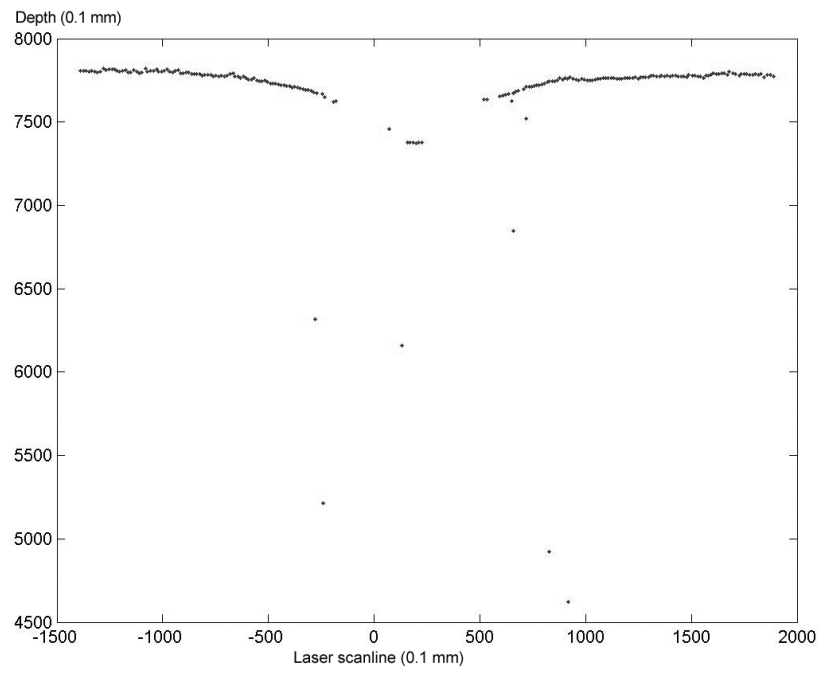

b)

Fig. 8 - Geometric profiles measured by the laser range-finder a) along the horizontal direction and b) along the vertical direction.

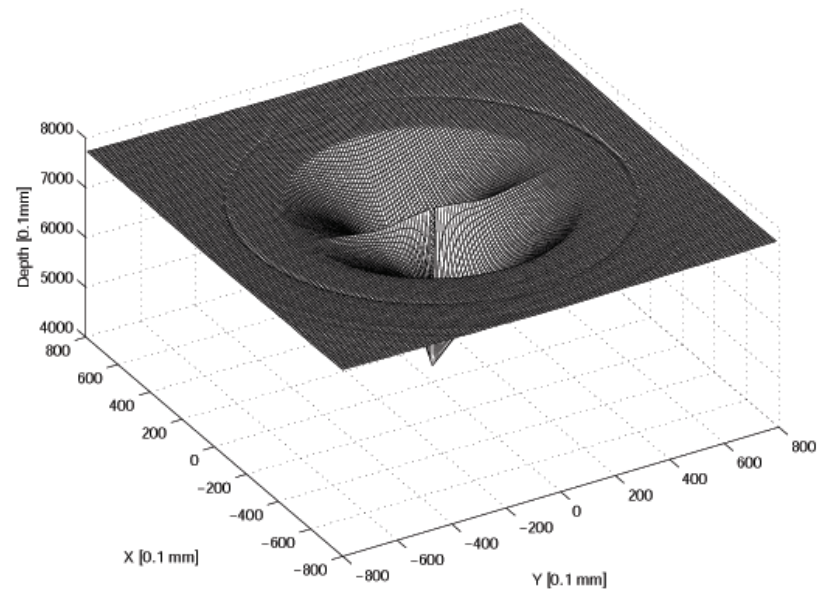

Fig. 9 - Surface map of the deformation resulting from the force applied by the probing rod. 
The resulting mapping serves as an input to a desktop hapto-visual human interface that allows a human teleoperator to experience the haptic feeling profiles at the point of contact as well as to see the image of a larger area around the point of contact on the explored object as captured by a video camera mounted on the robot manipulator. It includes a PHANTOM® $6 D O F[21]$ haptic device representing the handheld replica of the probing rod that provides the haptic feedback consisting of the 3D geometric coordinates of the point of contact measured by the laser range finder system and the force vector and torque components measured by the 6 DOF forcetorque sensor at the point of contact.

\section{MODELLING THE ELASTIC PROPERTIES OF 3D OBJECTS FROM EXPERIMENTAL TACTILE AND RANGE IMAGING DATA}

Many interactive virtual reality applications such as telemedicine or telelearning cannot rely on synthetic models of the manipulated objects and need models conformal to reality obtained from measurements of physical objects.

As high rendering rates are essential for the quality of the high-fidelity virtual environments [22], high-speed techniques are needed for the efficient storage, model transformation, and realtime rendering of large numbers of hapto-visual object models evolving in the virtual operation theater.

Neural networks $(\mathrm{NN})$ which are able to learn nonlinear behaviors from a limited set of measurement data can provide efficient and compact multi-media object modeling solutions. Due to their continuous, analog-like, memory behavior, NNs are able to provide instantaneously an estimation of the output value for input values that were not part of the initial training set. NNs consisting of a collection of simple neuron circuits provide the massive computational parallelism allowing for high rendering rates of complex models [23].

A NN modeling technique, Fig. 10, has recently been proposed by Cretu et al., [19], to map elastic behavior from data collected using a joint sensing strategy, combining tactile probing and range imaging.

The force/torque sensor shown in Fig. 6 is used to measure the force components applied on the object and a laser range finder measures the deformation of the surface of the object under the given force. The laser profiling approach shown in Fig. 7 is used to collect 75 to 100 scans of the same area within a few seconds while the applied force is kept constant. Each geometric profile scan consists of 512 samples measured along a straight laser sweep lines on the object's surface. In order to filter out the measurement noise, the mean value of all deformation profiles obtained under any given force is computed. The resulting mean value profiles, profile $\left(f_{i}\right)$, are then saved for each magnitude of normal force, $f_{i}$, applied on the object and for each cluster of similar elasticity. This provides efficient means to cope with the noise in the range data.

The process is initially guided by vision to define regions where the object lies. Then starting from the resulting cloud of 3D data, a neural gas NN yields a reduced set of cluster points on the 3D object's surface which are relevant for the tactile probing [24]. For each cluster of similar elasticity, a feedforward NN with two input neurons ( $Y$ and $F$ ), 25 hidden neurons and one output neuron $(Z)$ is employed to learn the relation between the applied forces measured by the force-torque sensor and the corresponding geometric profiles measured by the range finder. One network is needed to model the elastic behavior of each cluster. Once trained, the $\mathrm{NN}$ takes as inputs the position along the profile, $Y$, and the force, $F$, and outputs the surface deformation coordinate, $Z$, with respect to the unloaded surface location, as illustrated in Fig. 11 and Fig. 12.

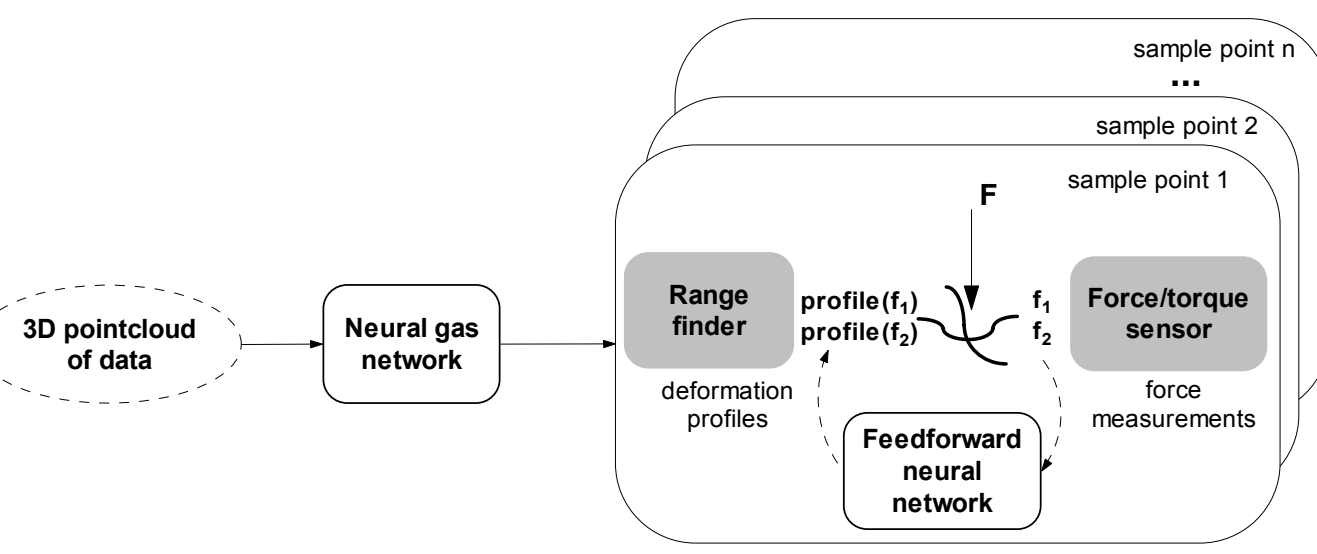

Fig. 10 - Neural network mapping and clustering of elastic behavior from tactile and range imaging data. 


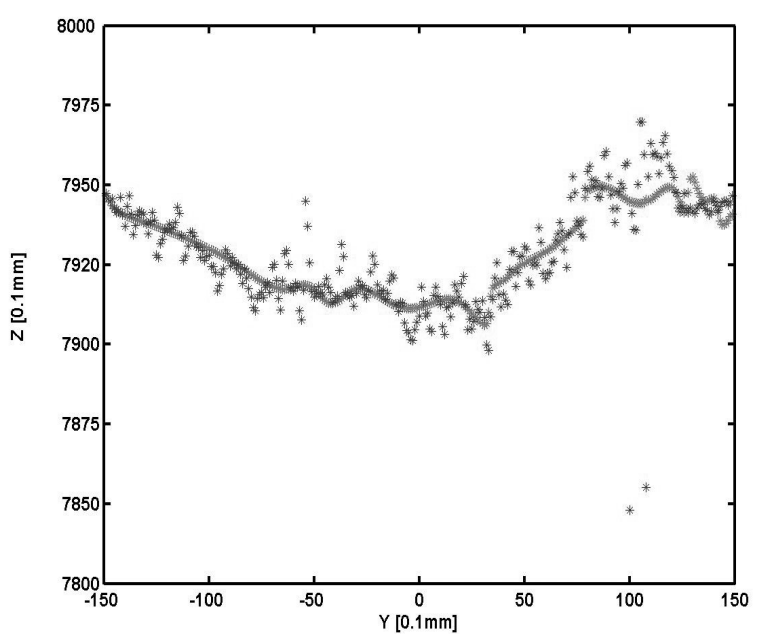

Fig. 11 - Real and NN modeled $(Y, Z)$ geometric profile of a semi-stiff material under a normal force $\mathrm{F}=\mathbf{0 . 3 7} \mathrm{N}$.

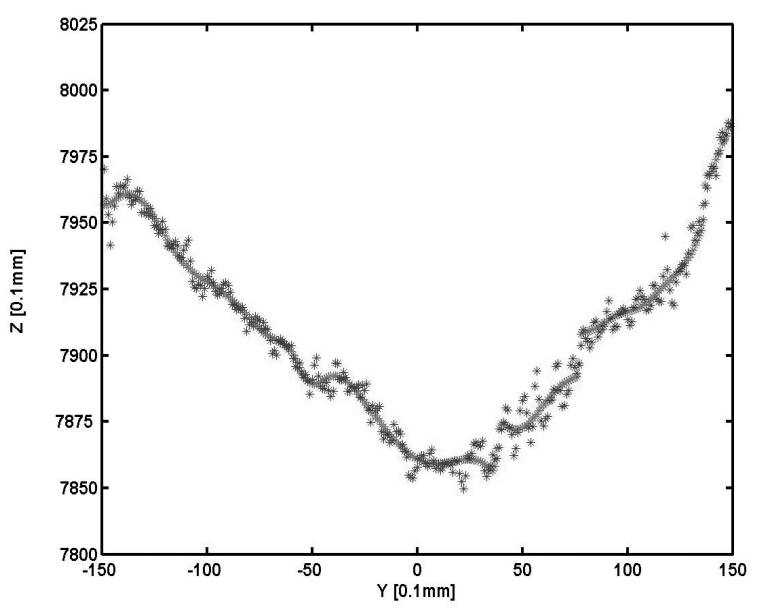

Fig. 12 - Real and NN modeled $(Y, Z)$ geometric profile of a semi-stiff material under a normal force $F=2.65 \mathrm{~N}$.

The feedforward NNs were trained for 10000 epochs using the Levenberg-Marquardt backpropagation algorithm with the learning rate set to 0.009 . The whole data set is used for training in order to provide enough samples. For the semi-stiff material, the mean square error reached during training is $3.50 \times 10^{-7}$.

The resulting hapto-visual $\mathrm{NN}$ model allows not only to recover the elastic parameters in the sampled points but also provides an estimate on the elastic behavior on surrounding points that are not part of the selected sampling point set. This model can be used in combination with the hapto-visual human interface, in place of the classical strain-stress mapping. This provides the interface with complex nonlinear features that cannot be accurately represented with standard linear models.

\section{ACKNOWLEDGMENT}

This work was funded in part by the Canadian Space Agency, the Natural Sciences and Engineering Research Council of Canada, the Communications and Information Technology Ontario, and the Materials and Manufacturing Ontario. Support from the Ontario Graduate Scholarship program is also acknowledged.

\section{REFERENCES}

[1] R. Sekuler, R. Balke, Perception, 2nd edition, McGraw-Hill, NY, 1990, Chapter 11. Touch, pp. 357-383.

[2] S.J. Lederman, D.T. Pawluck, "Lessons From the Study of Biological Touch for Robotic Haptic Sensing," in Advanced Tactile Sensing for Robotics (H.R. Nicholls, ed.), World Scientific, 1992.

[3] P.C. Bennett, R.J. Anderson, Robotic Mobile Manipulation Experiments at the US Army Maneuver Support Center, Sandia Report, SAND2002-1779, June 2002.

[4] P. Boulanger, M. Benitez, "A Tele Immersive System for Collaborative Artistic Creation," Proc. AVIR 2003 Conf., pp. 12-20, Geneva, Switzerland, Nov. 2003.

[5] K. Vlachos, E. Papadopoulos, D.N. Mitropoulos, "Design and Implementation of a Haptic Device for Training in Urological Operations," IEEE Trans. Robot. Automat., Vol. 19, No. 5, pp. 801809, 2003.

[6] A.M. Okamura, N. Smaby, M.R. Cutosky, "An Overview of Dexterous Manipulation," Proc. ICRA 2000 - IEEE Intl. Conf. on Robot. Autom., pp. 255-262, San Francisco, CA, Apr. 2000.

[7] X. Di, B.K. Ghosh. X. Ning, T.J. Tarn, " SensorBased Hybrid Position/Force Control of a Robot Manipulator in an Uncalibrated Environment," IEEE Trans. Contr. Syst. Technol., Vol. 8, No. 4, $635-645,2000$.

[8] G. Burdea, Ph. Coiffet, Virtual Reality Technology (2nd edition), Wiley, NJ, 2003.

[9] "Hand Technical Specification," Shadow Robot Company, London, England, http://www.shadowrobot.com/hand/overview.sht $m l$

[10] "Design of a Dextrous Hand for advanced CLAWAR applications," Shadow Robot Company, London, England, 2003, http://www.shadowrobot.com/downloads/dextro us_hand_final.pdf

[11] M.J. McDonald, Active Research Topics in Human Machine Interfaces, Sandia Report, SAND2000-2779, Dec. 2000.

[12] M. Benali-Khoudja, M. Hafez, J.-M. Alexandre, A. Kheddar, "Tactile interfaces: A state-of-the 
art Survey," Proc. ISR 2004 -35th Intl. Symp. Robotics, Paris, March 2004.

[13]R.W. Harrigan, P.C. Bennett, Dexterous Manipulation: Making Remote Manipulators Easy to Use, Sandia Report, SAND2001-3622, Nov. 2001.

[14]E.M. Petriu, D.C. Petriu, V. Cretu, "Control System for an Interactive Programmable Robot," Proc. CNETAC Nat. Conf. Electronics, Telecommunications, Control, and Computers, pp. 227-235, Bucharest, Romania, Nov. 1982.

[15]E.M. Petriu, W.S. McMath, "Tactile Operator Interface for Semi-autonomous Robotic Applications," Proc. Int. Symposium on Artificial Intell. Robotics Automation in Space, i-SAIRS'92, pp. 77-82, Toulouse, France, 1992.

[16] E.M. Petriu, W.S. McMath, S.K. Yeung, N. Trif, "Active Tactile Perception of Object Surface Geometric Profiles," IEEE Trans. Instrum. Meas., Vol. 41, No. 1, pp. 87-92, 1992.

[17]P. Payeur, C. Pasca, A.-M. Cretu, E.M. Petriu, "Intelligent Haptic Sensor System for Robotic Manipulation," IEEE Trans. Instrum. Meas., Vol. 55, No 4, pp. 1148-1159, August 2006.

[18] S.K. Yeung, E.M. Petriu, W.S. McMath, D.C. Petriu, "High Sampling Resolution Tactile Sensor for Object Recognition," IEEE Trans. Instrum. Meas., Vol. 43, No. 2, pp. 277-282, 1994.

[19]A.-M. Cretu, E.M. Petriu, P. Payeur, "Neural Network Mapping and Clustering of Elastic Behavior from Tactile and Range Imaging for Virtualized Reality Applications," Proc. IST 2006, IEEE Intl. Workshop on Imaging Systems and Techniques, pp. 17 - 22, Minori, Italy, April 2006.

[20] Installation and Operations Manual for StandAlone F/T Sensor Systems, ATI Industrial Automation Inc, Garner, NC, 1997, http://www.ati-ia.com

[21]PHANTOM Premium 1.5/6DOF, 1.5 HighForce/6DOF and 3.0/6DOF Haptic Devices, $\quad h t t p: / / w w w . s e n s a b l e . c o m / h a p t i c-$ phantom-premium-6dof.htm

[22]M. Mahvash, V. Hayward, "High-Fidelity Passive Force-Reflecting Virtual Environments," IEEE Trans. Robotics," Vol. 21, No. 1, pp. 38-46, 2005.

[23]E.M. Petriu, "Neural Networks for Measurement and Instrumentation in Virtual Environments," in Neural Networks for Instrumentation, Measurement and Related Industrial Applications, (S. Ablameyko, L. Goras, M. Gori, V. Piuri - Eds.), NATO Science Series, Computer and System Sciences Vol. 185, pp. 273-290, IOS Press, 2003.
[24] A.-M. Cretu, J. Lang, E.M. Petriu, "Adaptive Acquisition of Virtualized Deformable Objects with a Neural Gas Network", Proc. IEEE Int. Workshop on Haptic, Audio-Visual Environments and their Applications, pp. 165170, Ottawa, ON, Oct. 2005.

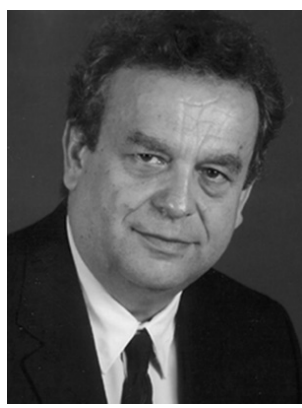

Emil M. Petriu is a Professor and University Research Chair in the School of Information Technology and Engineering at the University of Ottawa, Canada. Dr. Petriu's research interests include robot sensing and perception, intelligent sensors, interactive virtual environments, soft computing, digital integrated circuit testing. During his career he has published more than 200 technical papers, authored two books, edited other two books, and received two patents. He is a Fellow of IEEE, Fellow of the Canadian Academy of engineering, and Fellow of the Engineering Institute of Canada. He is a co-recipient of the IEEE's Donald G. Fink Prize Paper Award for 2003, and recipient of the 2003 IEEE Instrumentation and Measurement Society Award. He is also an Associate Editor of the IEEE Transactions on Instrumentation and Measurement and member of the Editorial Board of the IEEE I\&M Magazine.

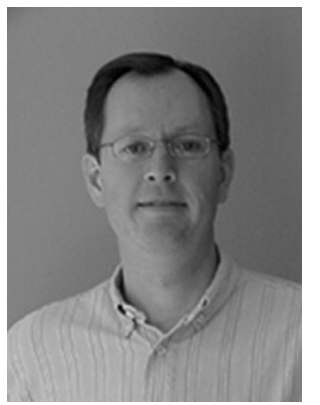

Pierre Payeur received the Ph.D. degree in Electrical Engineering from Laval University, Canada. In 1998, he cofounded the Vision, Imaging, Video and Autonomous Systems Research Laboratory (VIVA) at the University of Ottawa, Canada, where he is currently an Associate Professor in the School of Information Technology and Engineering. His research interests are in volumetric 3-D modeling, range imaging, tactile sensing, robot guidance, teleoperation and integration of computer vision in autonomous systems.

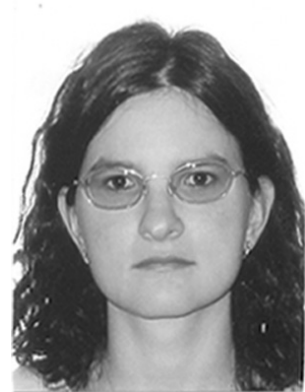

Ana-Maria Cretu obtained her Master degree from the School of Information Technology and Engineering at University of Ottawa, Canada, where she is now a Ph.D. candidate. Ms. Cretu's research interests include neural networks, tactile sensing, 3D object modeling, and multi-sensor data fusion. 